\title{
1 Preparation of Perfusive Chromatographic Materials 2 via Shear-Induced Reactive Gelation
}

3

4 Alberto Cingolani, Daniel Baur, Stefano Caimi, Giuseppe Storti and Massimo 5 Morbidelli*

6

7

8 Institute for Chemical and Bioengineering, Department of Chemistry and Applied Bioscience

9 ETH Zurich, Vladimir-Prelog-Weg 1-5/10, 8093 Zürich, Switzerland

$11 *$ corresponding author:

Prof. Dr. Massimo Morbidelli

HCI F 129

Vladimir-Prelog-Weg 1-5/10

8093 Zürich

Switzerland

Tel: +41446323034

E-mail: massimo.morbidelli@chem.ethz.ch

19 


\section{Abstract}

2 A simple method for producing highly porous materials suitable for chromatographic 3 applications is discussed. Starting from a dispersion of polymer core-shell nanoparticles (latex), 4 micrometer sized clusters $(100 \mu \mathrm{m})$ are produced via shear-induced Reactive Gelation. Thanks 5 to their fractal structure, these aggregates exhibit highly porous structures, with uniform pore size distribution ranging from 0.1 to several micrometers. The effect of different properties of

Keywords: Reactive Gelation, Macro-porous particles, Perfusive materials, polymer nanoparticles 


\section{Introduction}

Porous polymers are widely employed in a variety of different applications including separation and purification techniques [1,2], catalysis [3], thermal insulation [4] and medical tissue engineering [5]. Depending on the amount, morphology and mechanical characteristics of the desired porous material, different manufacturing methods are currently in use [6]. For example polymer-based materials with large pores are produced by suspension polymerization with the aid of a pore-generating system, mainly in the form of a porogen [6]. The resulting process involves multiple steps taking place at the same time, thus making the control of the final pore architecture very challenging. Moreover, the porogen is essential for the pore formation but needs to be fully extracted after the reaction, which may be difficult in some cases. Despite the extensive use of this approach, a reliable and comprehensive relationship between the reaction recipe and the morphological properties of the final material is far from being fully established [7-9]. Reactive Gelation, an alternative, porogen-free method for producing rigid macroporous polymer particles has been proposed previously by Marti et al. [10] and it enables the preparation of these materials in a closely controlled and step-wise manner. Starting from a stable colloidal suspension of polymer particles, additional monomer and initiator are added in order to swell them. These swollen particles, usually stabilized by ionic surfactants, are subsequently aggregated by destabilization induced in different ways: by screening the surface charges through the addition of electrolytes (Brownian aggregation), thus reducing the repulsive barrier among the particles, or by providing them enough kinetic energy to overcome the repulsive barrier (shear aggregation) [11]. Depending on the selected aggregation method, different products (e.g. monoliths or micrometer-size clusters) and different structures in terms of pore size distribution, have been obtained [11,12]. The last step of the Reactive Gelation consists in a post-polymerization of the previously added monomer/initiator mixture. This way 
the primary particles are more strongly bonded together, while retaining the porous structure and improving the mechanical strength and resistance of the final material.

Shear-induced aggregation is performed by exposing the latex to some kind of flow field. If applied without electrolyte addition, this mechanism is called pure shear-induced aggregation [13]. Owing to their fractal structure, the aggregates occupy more space while growing, which eventually leads to a percolation and the formation of a gel. It already has been shown [14] that a fraction of non-aggregated primary particles remains free when such a network is formed, which means that a fully percolating system can be obtained even when the aggregation is not complete. Xie et al.[15] showed that this process is affected by the size of both the primary particles and the resulting aggregates. It is also possible to carry out the aggregation process at large conversion while preserving the liquid-like behavior and limiting the system's viscosity increase [12]. This enables the use of continuous processes, which are easily scalable to large productivity. Moreover, the final dispersion of clusters and residual primary particles can be easily diluted by water addition, facilitating the subsequent steps of post-polymerization, wet filtration and packing. The final product can in fact be collected as ready-to-pack slurry of polymer particles in water.

The porous polymers produced in this work are specifically suited for chromatographic applications because they provide high transport efficiency [16]. As a matter of fact, a trade-off between pressure drop and separation performance is typically observed in chromatographic columns packed with conventional porous particles [2,16]. In contrast, particles and monoliths produced by Reactive Gelation exhibit pore sizes so large (radii larger than $200 \mathrm{~nm}$ ) that they permit convective flow through the particle, thus reducing (or even overcoming) the intraparticle diffusion limitations even at relatively large fluid velocities [17]. This allows using large particles, thus with low pressure drop and high flowrates, while avoiding mass transfer limitations. This behavior is known as perfusion and it enables flow-independent separation 
1 performance, which represents a great improvement towards the development of high-

2 throughput separation process[18-22]. Therefore, there is a great interest in the production of new stationary phases presenting such flow characteristics. As an example, the introduction of an additional set of very large pores in agarose beads (superporous agarose[23-26]), resulted in higher pore diffusivity and improved protein separation, in respect to conventional phases. Moreover, the same strategy, coupled this time with the very high mechanical resistance of crosslinked polystyrene[27], allowed the production of polymer-based materials able to perform separation at extremely high flow, still preserving high efficiency. On the other hand, the presence of huge pores might reduce the surface area, thus resulting in reduced binding capacity. This limitation may be overcome when brushes are grown from the pore surface [28] or nano-particles are used to cover the pore walls if they can be bound in a stable enough manner (e.g. by exploiting electrostatic interaction) [29]. In both cases, the effective surface area is increased, thus increasing the chromatographic performance.

In this work, a high-throughput method for producing highly porous and mechanically resistant polymer particles via reactive gelation is presented. The primary particles are aggregated through pure shear-induced aggregation by pumping the latex through a microfludizer, followed by post-polymerization. A comprehensive analysis of the effect of different features of the primary particles (architecture and size) on the morphology of the final material and its performance as chromatographic matrix is conducted, aiming at identifying the optimal operating conditions required to fulfill desired specifications. Pulse chromatography experiments using tracers of different sizes have been run on the produced materials and a comparison with commercially available polymer-based resins, both perfusive and nonperfusive, in nonretaining conditions, is presented.

\section{Experimental}




\section{$1 \quad 2.1$ Materials}

2 The following chemicals have been employed without further treatments: styrene (STY, Sigma

3 Aldrich, 4-ter-butylcatechol as stabilizer, purity $\geq 99 \mathrm{wt} \%$ ), divinylbenzene (DVB, Sigma

4 Aldrich, technical grade, $>80 \mathrm{wt} \%$ ), sodium dodecyl sulfate (SDS, Sigma Aldrich, BioUltra $\geq$

$599 \mathrm{wt} \%)$, potassium persulfate (KPS, Fluka, purity $\geq 99 \mathrm{wt} \%$ ), 2-2`-azo(2-methylpropinitirile)

6 (AIBN, Fluka, purity $\geq 98$ wt\%), sodium chloride (for analysis, Merck), sodium phosphate

7 dibasic, sodium phosphate monobasic (Sigma Aldrich). Dextran standards with different

8 molecular weights (Sigma Aldrich) have been used for packing testing. Ultra-pure grade water

9 has been prepared by Millipore Synergy (Millipore, Billerica, MA, U.S.A.). Deionized water to be used for the polymerization reactions has been de-oxygenated by degassing under vacuum and subsequent saturation with nitrogen. Commercial resins POROS ${ }^{\circledR} 50$ HS and Eshmuno ${ }^{\circledR}$ CPX have been purchased from ThermoFisher Scientific and Merck respectively and have been packed following the packing procedures provided by the manufacturers. The monolith $\mathrm{CIM}^{\circledR}$ r-Protein A DISK has been purchased from BIA Separations and used following the instructions of the producer.

\subsection{Synthesis of the Primary Particles}

The primary particles are produced via a semi-batch emulsion polymerization protocol. The procedure is divided into two steps: core and shell synthesis. In the first phase a hard, highly crosslinked (20 wt\%) seed of nanoparticles is produced. In the second step, a much softer, slightly crosslinked (1 wt\%) shell is grown around the previously formed particles. These two steps are done in series, thus feeding the monomer mixture required for the shell production directly after completion of the core synthesis. Several latexes have been produced with different particle architectures. Namely, the ratio between core and overall particle radii as well 
1

as the total particle sizes were varied. Details of all the recipes used in each synthesis are reported in Appendix A, while the applied general procedure is presented hereinafter.

\subsubsection{Core Synthesis}

A mixture of water and surfactant (SDS) is initially charged into the glass reactor and the temperature set to $70^{\circ} \mathrm{C}$ (Initial Charge, IC). Once this set-point is reached, a solution of water and initiator (KPS) is injected (Initiator Shot 1, IS1). In order to guarantee starved polymerization conditions, an emulsion of styrene, DVB, water, and surfactant is fed over the reaction time using an HPLC pump (Charged Feed 1, CF1). Moreover, a solution of water and KPS is continuously fed as well, as the total reaction time is longer than the half-life time of the initiator at the reaction temperature (Initiator Feed, IF). The conversion is frequently measured to ensure monomer-starved conditions.

\subsubsection{Shell Synthesis}

A new monomer solution (Charged Feed 2, CF2), this time composed only of styrene and DVB, is then fed to the system right after terminating the core synthesis in the same reactor. The previous initiator feed is disconnected, while a shot of water and KPS is added to keep the reaction running (Initiator Shot 2, IS2). Again, the conversion is monitored to ensure starved operation. After the new monomer addition is complete, the synthesis is left running in batch until full conversion is obtained.

\subsubsection{Latex Characterization}

During production, the latex is monitored in terms of particles size distribution (by dynamic light scattering (DLS) using a Zetasizer Nano ZS from Malvern) and monomer conversion (from the dry mass fraction of the sample)[30]. In the latter case, the latex is spread over quartz sand and analyzed at $120^{\circ} \mathrm{C}$ in air using a HG53 Moisture Analyzer from Mettler-Toledo. 


\section{$1 \quad 2.3$ Reactive Gelation}

2

3

4

5

6

\subsubsection{Latex Swelling}

Each latex is diluted with deionized water up to the desired dry mass fraction and swollen by an additional mixture made of divinylbenzene and oil-soluble initiator (AIBN, $5 \mathrm{wt} \%$ ). This mixture swells the outer layer of the particles almost exclusively, because the core is too highly crosslinked. The amount of added monomer is adjusted to be $30 \mathrm{wt} \%$ of the polymer mass in the particle shell. The obtained dispersion of swollen particles is then kept under stirring overnight to ensure complete equilibration.

\subsubsection{High Shear Destabilization}

The swollen latexes are then destabilized using the high shear device HC-5000 (Microfluidics, Westwood, MA, U.S.A.) equipped with a L30Z microchannel (rectangular cross section of $5.76 \times 10^{-8} \mathrm{~m}^{2}$, length of $5.8 \mathrm{~mm}$ ), referred to as "microchannel” in the following. The latex is pumped through it by a membrane pump and micrometer size clusters of primary particles are collected at the outlet. According to the specifications provided by the manufacturer, the generated shear rate $\dot{\gamma}(1 / \mathrm{s})$ is correlated to the pressure drop through the channel itself, $\Delta \mathrm{P}$, by the following empirical relationship:

$$
\dot{\gamma}=2.27 \times 10^{5} \cdot \Delta \mathrm{P}^{0.64}
$$

where $\Delta \mathrm{P}$ is the applied pressure drop in bar. This relationship is valid in a range of $\Delta \mathrm{P}$ values between 80 and 160 bar. In this work all samples have been aggregated using a constant $\Delta \mathrm{P}$ of 120 bar, corresponding to a flow rate in the channel of $918 \mathrm{~mL} / \mathrm{min}$, a residence time of 0.94 ms, and a shear rate of $4.8 \times 10^{6} 1 / \mathrm{s}$.

It is worth mentioning that the limiting factor of the setup to form aggregates is the operational limit of the pump. It was in fact observed experimentally that flooding occurs when a latex with 
too high dry solid content is passed through the microchannel, with head and piston housing clogged by polymer. Such clogging affects the process and the product quality significantly. Therefore, the dry solid content of the latexes was kept at $7.5 \mathrm{wt} \%$ and the suspension of aggregates leaving the microchannel is immediately diluted about ten times in deionized water and transferred into a three-neck flask. This way, the aggregates remain completely wet (preventing air contact) and fully segregated during the post-polymerization phase, thus avoiding the formation of polymer lumps. The aggregation extent (called "conversion" in the following) is measured by quantifying the mass fraction of aggregates with respect to the initial mass of polymer. This is done by precipitating the aggregates from the continuous phase by centrifugation and separating them from the residual primary particles (and very small aggregates), after which they are dried and weighted.

\subsubsection{Post-polymerization}

The clusters of primary particles produced by aggregation in the microchannel are kept together only by Van der Waals forces. In order to improve the mechanical properties of the aggregates, an additional polymerization step (called post-polymerization) is carried out taking advantage of the monomer added during the swelling step before the high-shear treatment. The three-neck flask containing the aggregates is immersed in an oil bath, connected to a reflux condenser, and kept under gentle stirring (100 rpm) and mild flow of nitrogen. Then, the temperature is raised to $70^{\circ} \mathrm{C}$, the nitrogen flow is stopped after one hour and the system is left reacting for at least twelve hours. Finally, the latex is first filtered with a $200 \mu \mathrm{m}$ mesh to remove possible large polymer lumps, and then dialyzed using a membrane with $10 \mu \mathrm{m}$ mesh for approximately half an hour to clear out residual primary particles.

\subsection{Packing}


1 The produced materials are slurry packed into GE Tricorn Columns of $50 \mathrm{~mm}$ length and $5 \mathrm{~mm}$

2 inner diameter. The applied packing procedure consists of housing the clusters in the column

3 and in the reservoir, and packing them in water with flow rates up to $9 \mathrm{ml} / \mathrm{min}$, which is the

4 maximum available flow rate of our pump. Materials that are not able to withstand such a high

5 flow rate are not considered of interest and discarded. The commercial resins are packed in the

6 same housings following the procedures reported by the supplier. Monoliths are acquired

7 prepacked and fitted in suitable housings.

\section{$8 \quad 2.5$ Product Characterization}

9 As the aggregates produced are in micrometer range, their size and internal structure is characterized by static light scattering (SLS). Samples are highly diluted and then analyzed using a Mastersizer 2000 (Malvern Instruments, Worcestershire, UK). The scattered light intensity can be used to evaluate the average structure factor $\langle S(q)\rangle$ of the aggregates, defined as[31]:

$$
\langle S(q)\rangle=\frac{I(q)}{I(0) P(q)}
$$

where $q$ is the magnitude of the scattering vector (defined as $\frac{4 \pi n}{\lambda} \sin \left(\frac{\theta}{2}\right)$, where $n$ is the refractive index of the continuous phase, $\lambda$ is the wavelength in vacuum, and $\theta$ is the scattering angle), $I(0)$ the scattered intensity at zero angle, $I(q)$ the angle-dependent scattered intensity and $P(q)$ the form factor of the primary particles measured using the same instrument. In order to relate the average structure factor to the radius of gyration, the Guinier approximation is used [31]:

$$
\langle S(q)\rangle=\exp \left(-\frac{q^{2}\left\langle R_{g}^{2}\right\rangle_{S(q)}}{3}\right) \quad\left(q\left\langle R_{g}\right\rangle_{S(q)}<1\right)
$$

where the root-mean square radius of gyration of the aggregates, $\left\langle R_{g}^{2}\right\rangle$, and the radius of gyration of the primary particles, $R_{g, p}$, are expressed as $\left\langle R_{g}^{2}\right\rangle=\left\langle R_{g}^{2}\right\rangle_{S(q)}+\left\langle R_{g, p}^{2}\right\rangle$ and $R_{g, p}=\sqrt{3 / 5} R_{p}$, 
respectively. Therefore, a rough evaluation of the average cluster size in terms of radius of gyration $(\langle R g\rangle)$ is obtained from the position of the bending of the curve in the double logarithmic plot. Note that the contribution of the primary particles is significant only for aggregates of small size and, therefore, it is not relevant in our case, where clusters with average size of several micrometers are produced and the residual primary particles have been removed. The same measurement technique provides additional information about the internal structure and the fractal dimension $\left(d_{f}\right)$ of the aggregates. Rayleigh-Debye-Gans theory may be applied where the power-law region of the average structure factor $\langle S(q)\rangle$ plotted as a function of $q$ on a double logarithmic plot yields a straight line whose slope is equal to $-d_{f}$, i.e., $\langle S(q)\rangle \propto$ $q^{-d_{f}}$. If clusters are compact enough, the particle size distribution can be derived as well by applying Mie theory, which assumes that the particles are spherical.

The overall surface area of the produced materials is evaluated by nitrogen adsorption measurements through the Brunauer-Emmett-Teller (BET) equation, using TriStar3000 equipment (Micrometrics, Norcross, GA, U.S.A.).

Pore size distribution and total porosity of the aggregates is measured by mercury intrusion porosimetry (equipment Pascal 140 and Pascal 440, Thermo Scientific, Waltham, MA, U.S.A.). In several samples, a clear distinction between internal and external porosity cannot be made in the measurements. Therefore, pores bigger than those intruded by Hg at atmospheric pressure (i.e., equal to or larger than $15 \mu \mathrm{m}$ according to the Washburn equation) are considered as external voids.

Visual inspection of the aggregates is performed by Scanning Electron Microscopy (Gemini 1530 FEG, Zeiss, Oberkochen, Germany).

Finally, direct characterization of the produced material, as well as of the commercial resins and monoliths is carried out by applying inverse size-exclusion chromatography (ISEC) of 
suitable tracers to the columns. Pulses of dextrans (polymerized anhydroglucose) in water (25mM, pH 7, phosphate buffer) of different molecular weights are injected at different flow rates to determine the total porosity and the maximum accessible pore size for each material after packing, as well as the dependence of the column efficiency on the flow rate in terms of height equivalent to the theoretical plate (HETP). All of the chromatographic analyses are run using a high performance liquid chromatography (HPLC) unit equipped with auto-sampler, refractive index detector, UV diode-array detector, and isocratic pump (Agilent Series 1100, Agilent Technologies, Santa Clara, CA, U.S.A.). The pressure drop of each column is also measured at different flow rates using the manometer of the HPLC pump.

\section{Results and Discussion}

\subsection{Production of Porous Materials}

Flowing through the microchannel the particles are provided with enough kinetic energy to overcome the energy barrier due to electrostatic repulsion[11]. Therefore in the high shear region of the channel, primary particles as well as already formed clusters undergo aggregation. Since the aggregation rate constant is proportional to the third power of the size of the colliding objects, the interaction between large objects is highly favoured [32]. At the same time, the largest clusters may break under the action of the hydrodynamic stress [33]. Eventually, a dynamic equilibrium between size increase by aggregation and reduction by breakage is established. Considering the swelling of the outer layer of the particles, which reduces the glass transition temperature of the polymer considerably [34], the particles within the clusters interpenetrate to different extents depending on the shell thickness [35]. This phenomenon has an effect on the aforementioned interplay between aggregation and breakage as well.

During the post-polymerization process, a strong interconnection is formed among the nanoparticles within a single cluster through the polymerization of the additional monomer 
present in the interpenetrating region of the neighboring particles. Their structure becomes mechanically more resistant and thus suitable for chromatographic applications. In the following only materials which could be packed in a column have been considered. Since many parameters, namely the core-shell particle architecture and the particle size, affect the resulting characteristics of the produced materials, different latexes, listed in Table 1, have been synthesized and characterized. The influence of each parameter on the properties of the final clusters is discussed in the following.

\subsection{Structure Characterization}

\subsubsection{Effect of Particle Architecture}

The clusters formed from latexes L80/110, L90/110 and L100/110 (different core-to-particle size ratio, same particle size) are considered first. Note that the core is highly crosslinked (20 wt\% DVB) and thus impenetrable to the swelling monomer. On the other hand, the monomer can easily penetrate the much softer shell (1 wt\% DVB).

Under the effect of shear (while passing through the microchannel), the polymer nano-particles are destabilized and aggregate in macroscopic clusters. The core-to-particle ratio has a clear influence on the extent of aggregation (conversion) and indeed the thicker the shell, the larger the number of clusters formed. The presence of a soft shell on the particles surface allows them to undergo a deeper interpenetration upon collision, making the aggregation events very efficient and almost irreversible. Indeed the same effect also increases the resistance to breakage (Figure 1). On the other hand, the residence time within the microchannel is not long enough to guarantee full aggregation and primary particles remain in all cases. Not surprisingly, the thicker the shell, the larger are the resulting aggregates, as illustrated in Figure 2, where the different cluster size distributions are reported. This conclusion reinforces the mechanism described previously, whereby the degree of interpenetration between the primary particles increases the mechanical stability of the aggregates. This is further supported by SEM images 
1 (see Figure 3), in which different interpenetration depths between different primary particles is

2 evident. While the primary particles are visible and recognizable in the case of clusters made

3 from L90/110 and L100/110, they almost lose their identity in the case of latex L80/110.

4 Moreover, the morphology in terms of fractal dimension is not affected much by the different

5 particle architecture and only small variations are visible. Indeed, a value of fractal dimension

6 of $2.5 \pm 0.1$ has been estimated for all samples by SLS analysis.

7

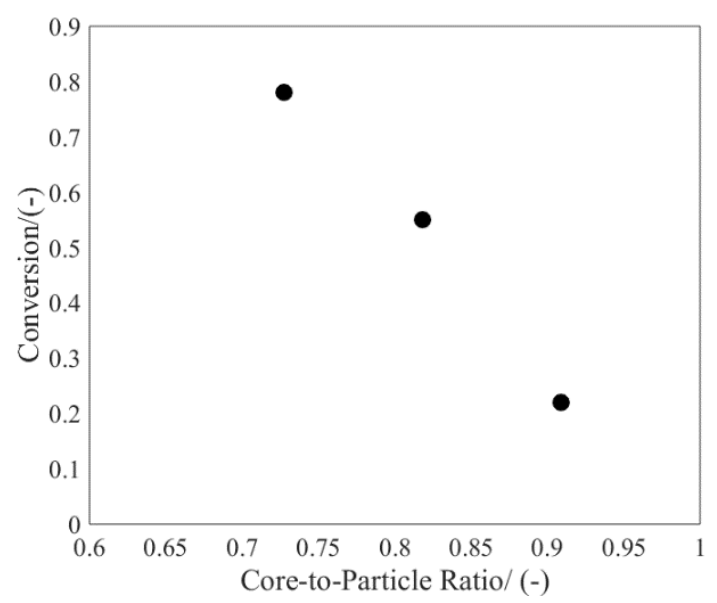

Figure 1: Aggregation extent (conversion) as a function of the core-to-particle ratio (same particle size, $110 \mathrm{~nm}$ )
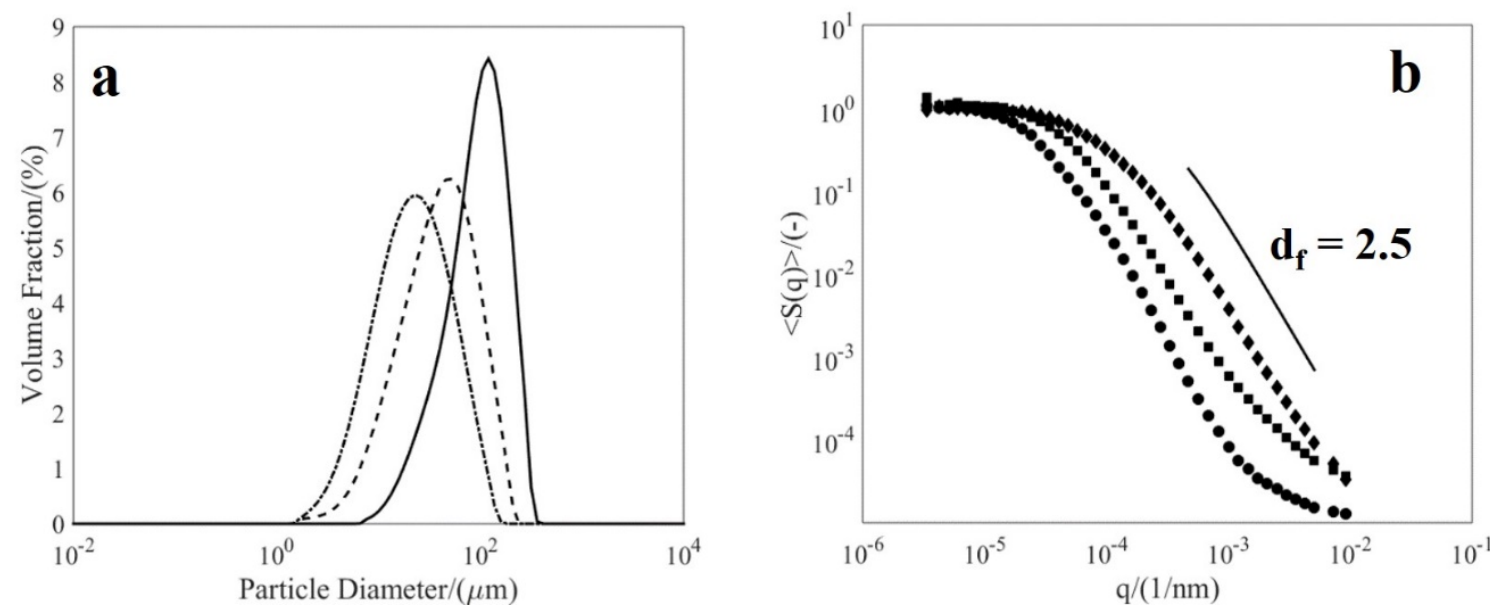

Figure 2: Particle size distribution (a) and structure factor curve (b) for clusters produced from particles with different architecture and constant size. Solid line and black dots for L80/110, dashed line and black squares for L90/110 and dotted line and black diamonds for L100/110 


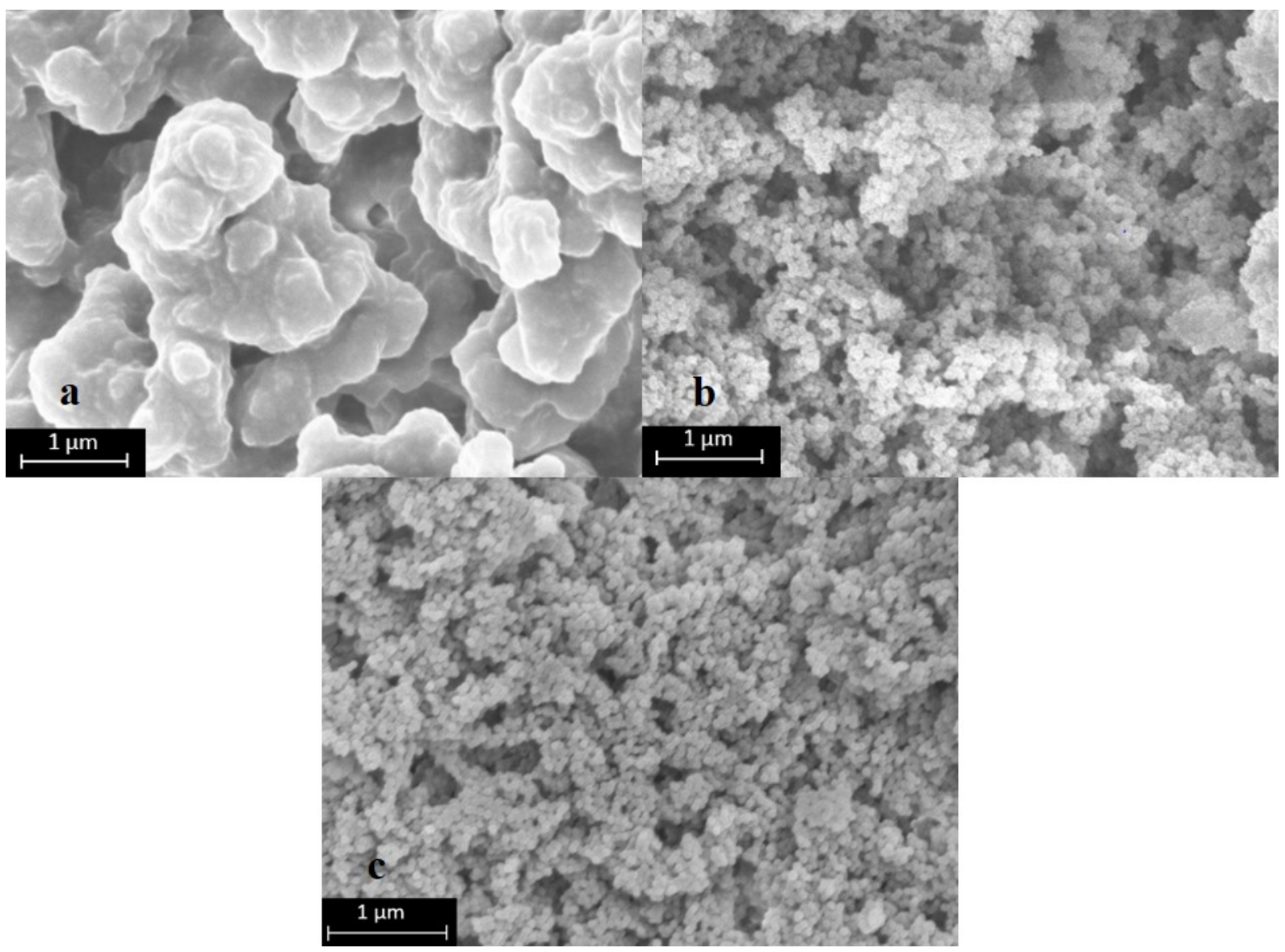

Figure 3: SEM pictures of the three different samples produced from L80/110 (a), L90/110 (b) and L100/110 (c)

The previously produced materials have been slurry packed and their ability to withstand the shear induced by a solvent flowing through at high velocity has been tested. Specifically, the maximum possible flow rate before the material collapses has been measured and the values are reported in Table 2. The failure of the matrix resulted into the formation of a compact polymer plug within the column and a dramatic increase in back pressure. In the case of the clusters produced from latex L80/110, no failure could be induced at all and the reported value of $9 \mathrm{~mL} / \mathrm{min}$ simply corresponds to the maximum flow rate of the pump used. This highlights again the strong role that the interpenetration among the particles, which is favored by the presence of a thick outer shell, plays in determining the mechanical strength of the resulting clusters.

\subsubsection{Effect of Particle Size}


1 In this section the clusters formed from latexes L80/110, L53/73 and L41/56 (core-to-size ratio

2 constant and equal to 0.73 , but different particle size) are investigated. The same architecture,

3 including crosslinked core (20 wt\% DVB) and much softer shell (1 wt\% DVB) is considered.

4 Figure 4 shows the conversion measured with respect to the primary particle size. As expected,

5 larger particles leads to higher extent of aggregation. The aggregation rate constant within the

6 microchannel is in fact proportional to the third power of the size of the colliding particles,

7 making the interaction between large objects highly favored [13]. This is in agreement with the

8 findings of Xie et al.[15], who showed already that a lower cluster volume fraction, and thus a

9 lower conversion, is required for smaller particles to achieve gelation at the outlet of the microchannel. About the morphology of the clusters, Figure 5 confirms that in fact the core-toparticle ratio is the parameter dominating the final characteristics of the materials. Clusters with very similar size and morphology in terms of fractal dimension are obtained when using primary particles of different size but identical architecture. This is because it is the high degree of interpenetration among the primary particles that makes the clusters more prone to aggregation and more resistant to breakage during the whole process.

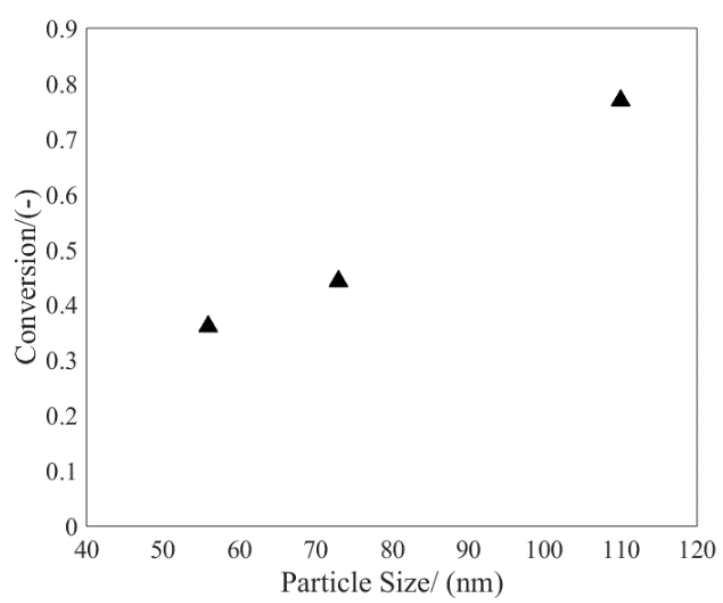




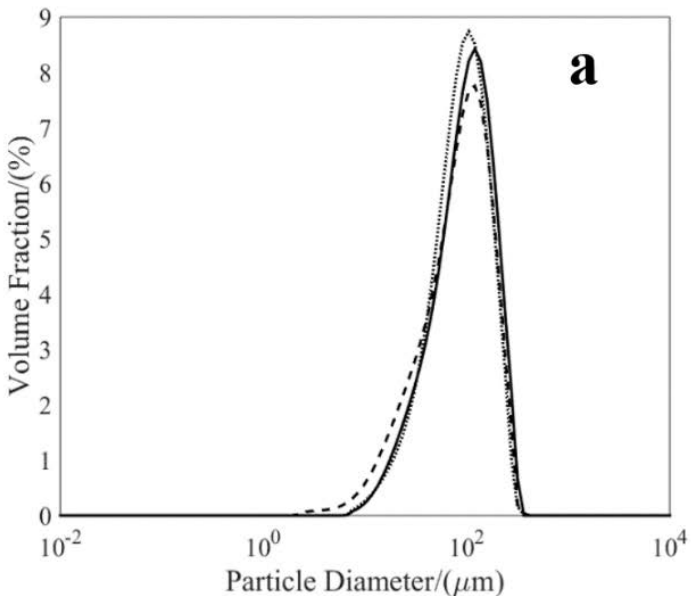

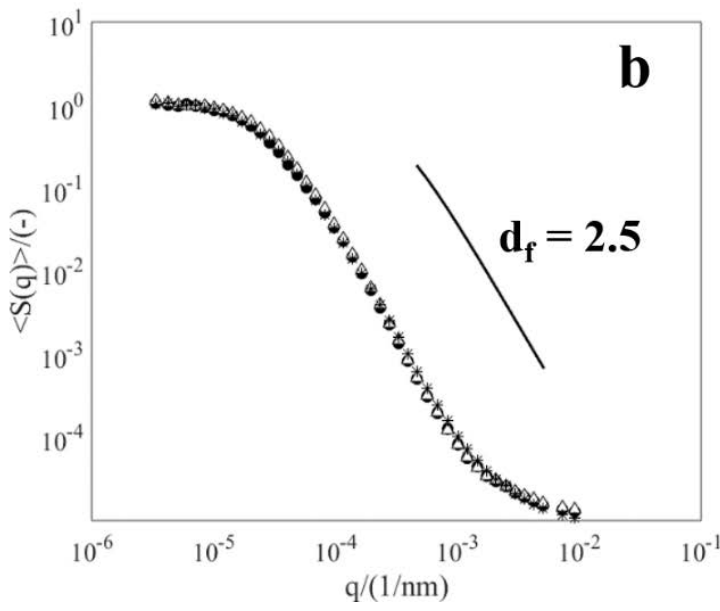

Figure 5: Particle size distribution (a) and structure factor curve (b) for clusters produced from particles with different size and same architecture. Solid line and black dots for L80/110, dashed line and white triangles for L53/73 and dotted line and asterisks for L41/56.

5

This second set of materials has also been slurry packed following the procedure previously described. Once housed in the column, all of these clusters were able to easily withstand a flow rates of up to $9 \mathrm{~mL} / \mathrm{min}$ without collapsing and with extremely low backpressure.

This is clearly due to the deep interpenetration among the primary particles which however also reduces the surface area. In Table 3 the values obtained by nitrogen physisorption are reported, showing that values equal to or below $10 \mathrm{~m}^{2} / \mathrm{g}$ are obtained in all cases. This means that such interpenetration closes most of the smaller pores, leaving only very large pores within the clusters. This result is also confirmed by the mercury intrusion curves reported in Figure 6 for all the samples. Evidently, the available porosity falls in the range between $0.1 \mu \mathrm{m}$ to several micrometers. Moreover, it can be noticed that the materials produced from L4156 present a shift towards slightly smaller pores, suggesting indeed that smaller initial particles generates smaller interstitial spaces within them. In light of these results, we can regard these packed materials in the column as a framework composed of very hard particles (after postpolymerization, the degree of crosslinking within the cluster domain is very high) with extremely large pores. Not surprisingly, such a system is able to withstand extremely high flow 
2 SEM pictures shown in Figure 7.

3

4

5

6
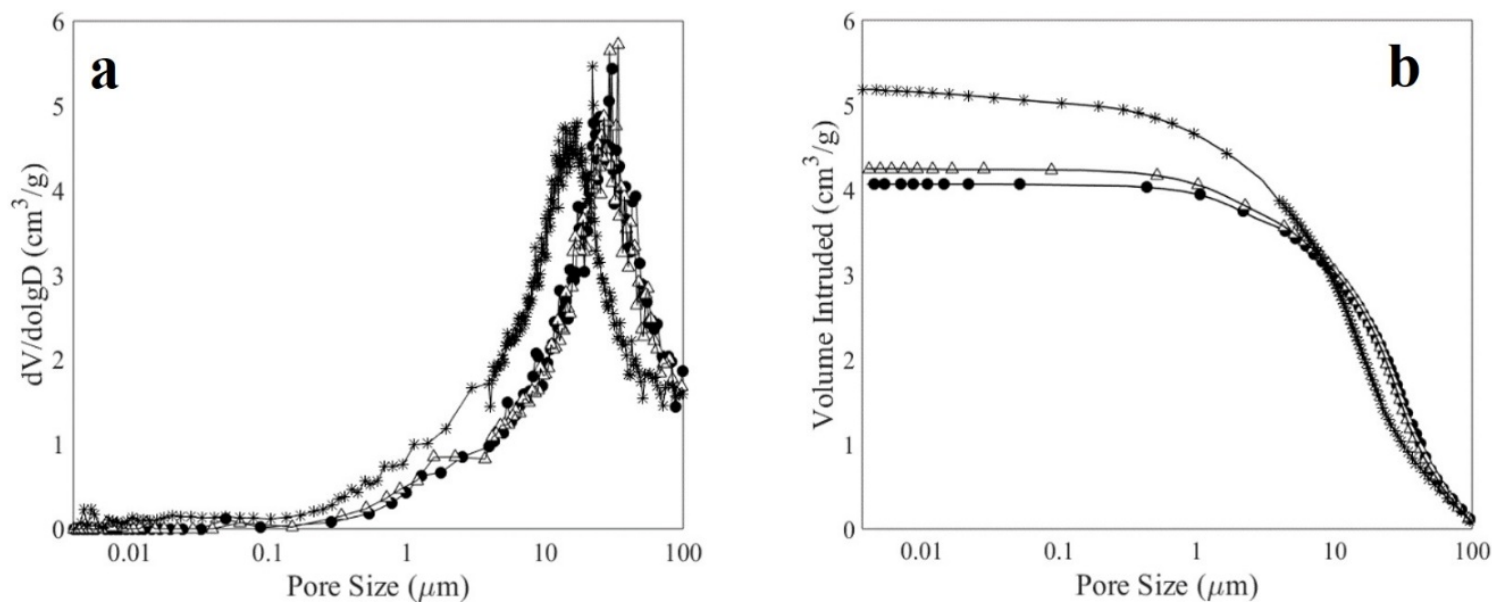

Figure 6: Differential (a) and cumulative (b) pore size distribution for the materials produced from latex L80/110 (black dots), L53/73 (white triangles) and L41/56 (asterisk).

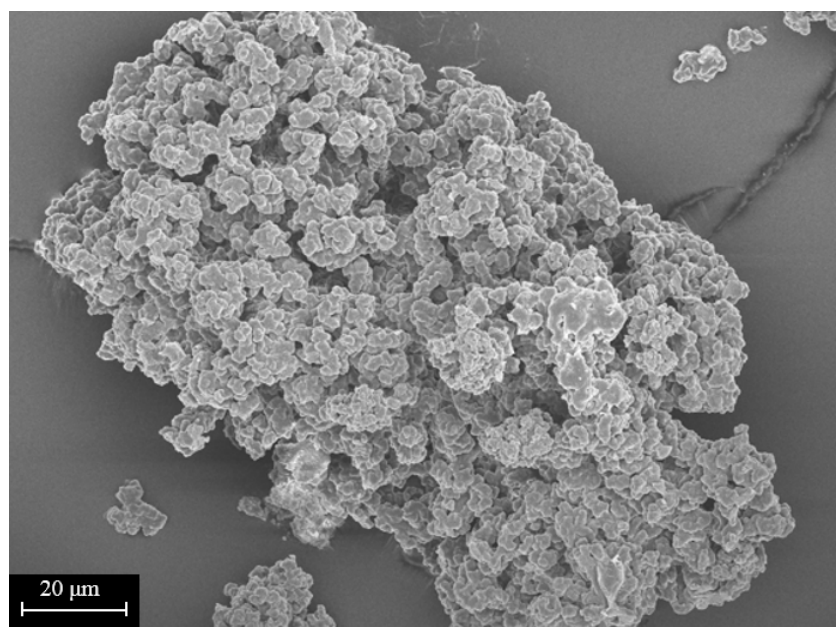

Figure 7: SEM picture of a cluster produced using the microchannel using latex L80/110

\subsection{Chromatographic Characterization}

\subsubsection{Packing Characteristics}


1 As already shown, the produced stationary phases that are able to withstand the applied packing

2 procedure have average particle sizes in the range of 100 micrometers in diameter and pore

3 sizes up to several micrometers. These characteristics make them considerably different from

4 popular commercial polymer-based materials, either that they are perfusive, such as POROS 50

5 HS, nonperfusive, such as Eshmuno CPX, or monolithic, such as CIM ${ }^{\circledR}$ r-Protein A DISK, as

6 can be seen from the comparison of average particle and pore sizes shown in Table 4 .

7 The pressure drop data shown in Figure 8 indicates that the commercial materials exhibit 8 considerably larger pressure drops. This difference can be imputed not only to the obvious 9 effect of the smaller particle size, but also to the lower fraction of very large pores.

In Figure 9, the column porosity for all the investigated stationary phases measured by SEC is shown as a function of tracer molecular weight (dextrans), which correlates with its hydrodynamic radius [36]. As expected, the porosity of the commercial materials decreases for bigger dextran species, because the smaller pores exclude larger molecules. This exclusion is not observed for our materials: indeed, they always exhibit constant value of porosity above 85\% independently of the tracer size. This is in agreement with the results of mercury intrusion measurements, which already showed the presence of very large pores and large void fractions in the clusters. This confirms that, compared to the commercial materials, the technology based on Reactive Gelation allows producing pore size distributions centered on larger average sizes, thus excluding the formation of smaller pores. 


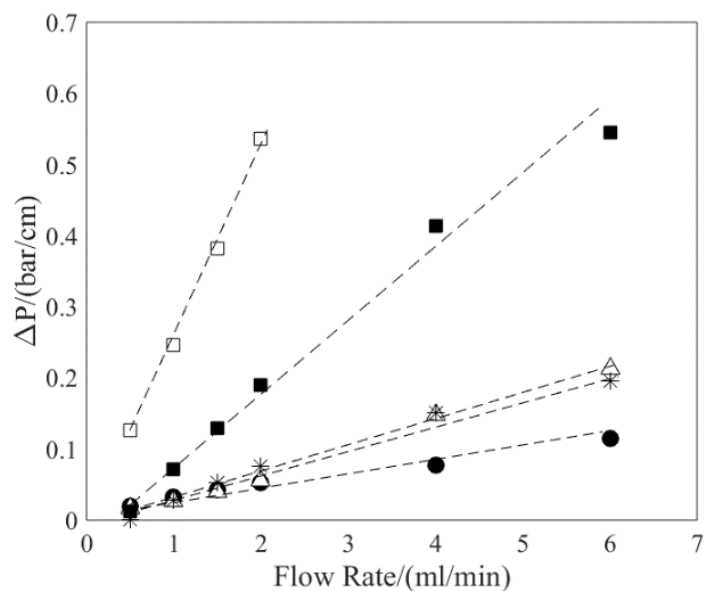

2 Figure 8: Pressure drops for all the packed materials: Eshmuno CPX (white squares), POROS 50 HS (black 3 squares), L80/110 (black dots), L53/73 (white triangles) and L41/56 (asterisks). Dashed lines are shown to guide 4 the eye.

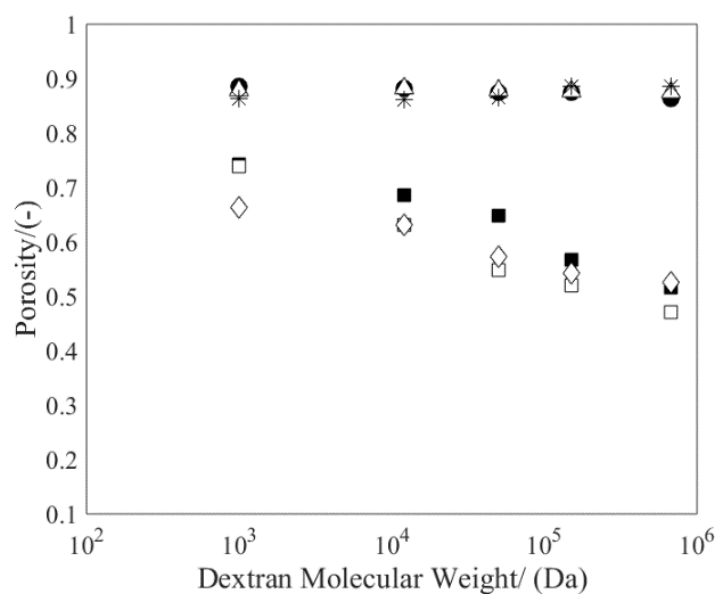

Figure 9: Porosity measured using tracers with different size for all the packed materials: Eshmuno CPX (white squares), POROS 50 HS (black squares), CIM ${ }^{\circledR}$ r-Protein A DISK (white diamonds) L80/110 (black dots), L53/73 (white triangles) and L41/56 (asterisks)

\subsubsection{Elution Performance}

The HETP values of each of the packed columns have been measured using tracers at different molecular size under non-adsorbing conditions. In particular, dextrans with molecular weights of $12 \mathrm{kDa}(\mathrm{Dex} 12000)$ and $150 \mathrm{kDa}(\mathrm{Dex} 150000)$ have been chosen as representative of typical sizes of large biomolecules (2.9 $\mathrm{nm}$ and $9 \mathrm{~nm}$ of hydrodynamic radius, respectively), the purification of which is one of the main applications of preparative chromatography in industry. 
1 As shown in Figure 10a, the HETP values of both Eshmuno CPX and POROS 50 HS increase

2 with the flow rate for the smallest species (Dex12000), meaning that the column becomes less

3 efficient at larger flow rates. On the other hand, all our materials exhibit HETP values almost

4 constant with the flow rate, a behavior very comparable to that of the monolith CIM ${ }^{\circledR}$ r-Protein

5 A DISK. Even though the efficiency of the monolith remains larger, the flow-independent

6 behavior of our packings is quite remarkable. Aware of the difficulties that can arise in the full

7 description of convective mass transfer[37], it is anyhow possible to notice that our materials

8 show an extremely perfusive characteristic. In the case of the largest tracer (Dex150000), Figure

9 10b shows increasing HETP values at increasing flow rates for Eshmuno CPX, whereas some flattening is observable for POROS $50 \mathrm{HS}$, given the perfusive nature of that material. Once again, all our materials all behave as the monolith, showing a flat HETP profile for all considered flowrates.

Although the measured HETP values are smaller for the monolith than for our materials, the latter offer the key advantage of an easy packing into conventional columns, without the requirement of special housings. This aspect represents a major advantage in terms of versatility and broadens the range of possible applications of materials produced thorough shear-induced reactive gelation. 

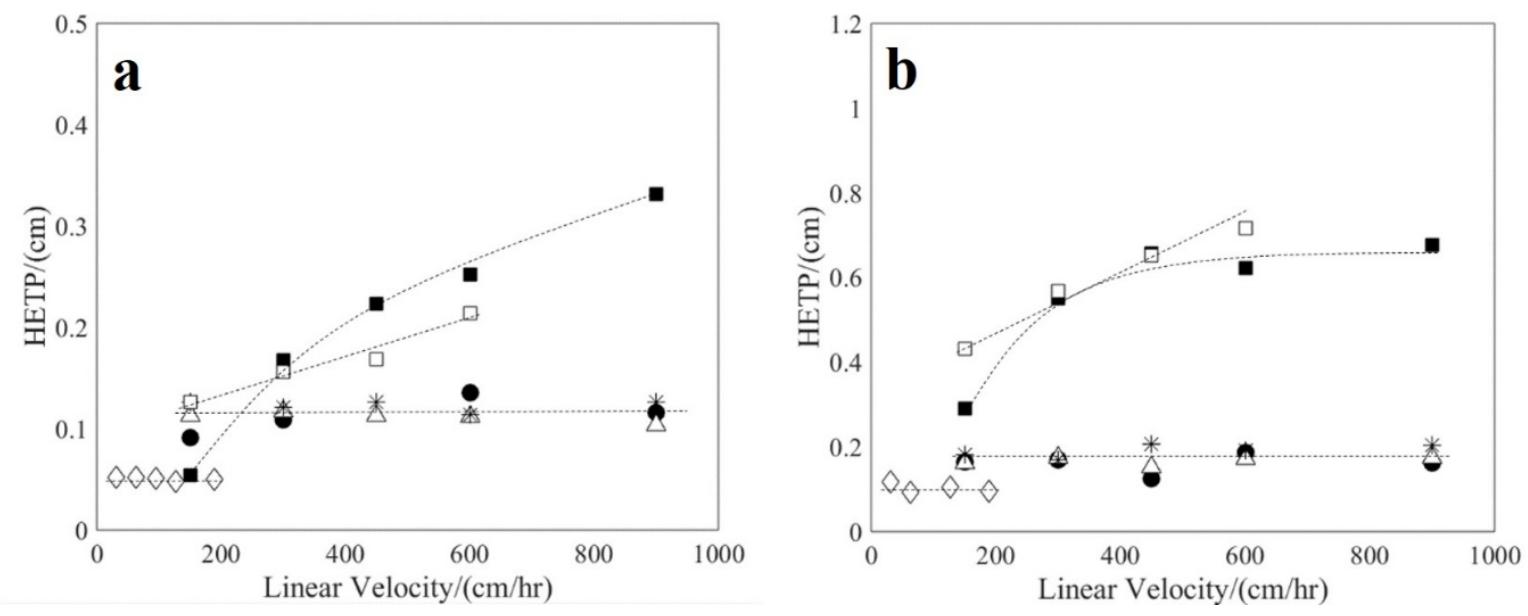

Figure 10: HETP as a function of flow rate for the species Dex12000 (a) and Dex150000 (b): Eshmuno CPX (white squares), POROS 50 HS (black squares), CIM ${ }^{\circledR}$ r-Protein A DISK (white diamonds) L80/110 (black dots), L53/73 (white triangles) and L41/56 (asterisks). Dashed curves are shown to guide the eye.

\section{Conclusion}

In this work, the effects of different properties of the primary particles on the final properties of porous clusters produced by Reactive Gelation under shear have been investigated. In particular, the effects of core-to-particle ratio and particle size have been explored. All the produced materials have been tested as potential chromatographic packings by measuring pressure drops and column efficiencies (HETP) after packing.

While the particle size was found to have little impact, the core-shell architecture of the primary particles affects the size of the produced aggregates and their mechanical resistance to a large extent. As a matter of fact, a small core-to-particle ratio is required to achieve highly porous materials able to withstand high flow rates after packing with small pressure drops.

Considering the chromatographic efficiency of columns packed with these materials, their nature enables flow rate independent HETP values. This achievement is a consequence of the large pore size, up to several micrometers, which makes the contribution of convective flow through the clusters dominate the mass transfer. Notably, flat HETP profiles resembling those 
1 typical of monolithic packings have been observed when large dextran tracers have been eluted.

2 Moreover, comparison with similar commercial polymer-based packings shows that shear-

3 induced Reactive Gelation produces porous materials with better pressure drop and mass

4 transfer properties, particularly with respect to their perfusion characteristics, that is HETP

5 values which remain constant for increasing fluid velocity. These consideration makes the

6 materials produced in this work extremely promising as efficient and easy-to-pack base

7 scaffolds suitable for further functionalization. This last step will make them specific to the

8 required separation target and partly compensate for the reduced specific area associated with

9 the large pore sizes. 


\section{Acknowledgments}

2 First of all the authors would like to thank Baptiste Jaquet for the great help with the

3 microchannel usage. Secondly the authors are really thankful to Annette Röthlisberger and

4 Michael Plötze for the help with the Mercury Intrusion measurements. Moreover, they would

5 like to thank Anna Beltzung and Antoine Klaue for the help with the BET measurements and

6 the SEM pictures respectively. Last but not least they express their gratitude to Dr. Hua Wu,

$7 \quad$ Nicole Ulmer and Ruben Wälchli for helpful discussion.

\section{$8 \quad$ Funding}

9 This research did not receive any specific grant from funding agencies in the public, commercial or not-profit sectors. 


\section{References}

2 [1] G. Carta, A. Jungbauer, Downstream Processing of Biotechnology Products, 2010. doi:10.1002/9783527630158.ch1.

[2] G. Guiochon, Basic Principles of Chromatography, in: Ulmann’s Enciyclopedia Ind. Chem., -VCH, Wiley, 2012. doi:10.1002/14356007.b05_155.pub2.

6 [3] D.C. Sherrington, Preparation, structure and morphology of polymer supports, Chem. Commun. (1998) 2275-2286. doi:10.1039/a803757d.

[4] G.-F. Chen, L. Peisheng, Applications of Polymer Foams, in: Butterworth-Heinemann (Ed.), Porous Mater. Process. Appl., 2014: pp. 383-410. doi:10.1016/B978-0-12407788-1.00008-3.

[5] H.P. Hentze, M. Antonietti, Porous polymers and resins for biotechnological and

[6] F.E. Gokmen, M. Talha; Du Prez, Porous polymer particles-A comprehensive guide to synthesis, characterization, functionalization and applications, Prog. Polym. Sci. 37 (2012) 365-405.

[7] F. Svec, Porous polymer monoliths: Amazingly wide variety of techniques enabling their preparation, J. Chromatogr. A. 1217 (2010) 902-924. doi:10.1016/j.chroma.2009.09.073.

[8] F. Svec, J.M.J. Frechet, Kinetic Control of Pore Formation in Macroporous Polymers. Formation of “Molded” Porous Materials with High Flow Characteristics for Separations or Catalysis, Chem. Mater. 7 (1995) 707-715. doi:10.1021/cm00052a016.

9] Malia, F. Svec, J.M.J. Frechet, Monodisperse polymer beads as packing material for 
high-performance liquid chromatography: Effect of divinylbenzene content on the porous and chromatographic properties of poly(styrene-co-divinylbenzene) beads prepared in presence of linear polystyrene as, J. Polym. Sci. Part A Polym. Chem. 32 (1994) 2169-2175. doi:10.1002/pola.1994.080321120.

[10] N. Marti, F. Quattrini, A. Butté, M. Morbidelli, Production of Polymeric Materials with Controlled Pore Structure: the Reactive Gelation’’ Process, Macromol. Mater. Eng. 290 (2005) 221-229. doi:10.1002/mame.200400344.

[11] H. Wu, M. Morbidelli, Gelation of polymeric nanoparticles, Particuology. 14 (2014) 111. http://www.elsevier.com/authorsrights.

[12] B. Brand, M. Morbidelli, M. Soos, Shear-Induced Reactive Gelation, Langmuir. 31 (2015) 12727-12735.

[13] A. Zaccone, D. Gentili, H. Wu, M. Morbidelli, E. Del Gado, Shear-Driven Solidification of Dilute Colloidal Suspensions, Phys. Rev. Lett. 106 (2011) 138301. doi:10.1103/PhysRevLett.106.138301.

[14] H. Wu, A. Tsoutsoura, M. Lattuada, A. Zaccone, M. Morbidelli, Effect of Temperature on High Shear-Induced Gelation of Charge-Stabilized Colloids without Adding Electrolytes, Langmuir. 26 (2010) 2761-2768. doi:10.1021/la902800x.

[15] D. Xie, H. Wu, A. Zaccone, L. Braun, H. Chen, M. Morbidelli, Criticality for shearinduced gelation of charge-stabilized colloids, Soft Matter. 6 (2010) 2692-2698. doi:10.1039/c002043e.

[16] B. Coquebert De Neuville, A. Lamprou, M. Morbidelli, M. Soos, Perfusive ionexchange chromatographic materials with high capacity, J. Chromatogr. A. 1374 (2014) 180-188. doi:10.1016/j.chroma.2014.11.066.

[17] G. Storti, M. Morbidelli, M. Soos, A. Lamprou, B. Brand, Method for the preparation 
of macroporous particles and macroporous particles obtained using such a method, WO 2014079580 A1, 2014.

[18] G. Carta, A.E. Rodrigues, Diffusion and convection in chromatographic processes using permeable supports with a bidisperse pore structure, Chem. Eng. Sci. 48 (1993) 3927-3935. doi:10.1016/0009-2509(93)80371-V.

[19] D. Whitney, M. Mccoy, N. Gordon, N. Afeyan, Characterization of large-pore polymeric supports for use in perfusion biochromatography, J. Chromatogr. A. 807 (1998) 165-184. https://doi.org/10.1016/S0021-9673(98)00068-5.

[20] N.B. Afeyan, N.F. Gordon, I. Mazsaroff, L. Varady, S.P. Fulton, Y.B. Yang, F.E. Regnier, Flow-through particles for the high-performance liquid chromatographic separation of biomolecules: perfusion chromatography, J. Chromatogr. A. 519 (1990) 1-29. doi:10.1016/0021-9673(90)85132-F.

[21] M. McCoy, K. Kalghatgi, F.E. Regnier, N. Afeyan, Perfusion chromatographycharacterization of column packings for chromatography of proteins, J. Chromatogr. A. 743 (1996) 221-229. https://doi.org/10.1016/0021-9673(96)00371-8.

[22] N.B. Afeyan, S.P. Fulton, F.E. Regnier, Perfusion chromatography packing materials for proteins and peptides, J. Chromatogr. A. 544 (1991) 267-279. doi:10.1016/S00219673(01)83991-1.

[23] P.-E. Gustavsson, P.-O. Larsson, Superporous agarose, a new material for chromatography, J. Chromatogr. A. 734 (1996) 231-240. doi:10.1016/00219673(95)01304-0.

[24] Q.H. Shi, X. Zhou, Y. Sun, A novel superporous agarose medium for high-speed protein chromatography, Biotechnol. Bioeng. 92 (2005) 643-651. doi:10.1002/bit.20652. 
1 [25] Y. Sun, G.Y. Sun, Z. Yang, X.Y. Dong, Biporous polymeric beads fabricated by double emulsification for high-speed protein chromatography, J. Appl. Polym. Sci. 103 (2007) 17-23. doi:10.1002/app.23872.

[26] X.Y. Jiang, S. Bai, Y. Sun, Fabrication and characterization of rigid magnetic monodisperse microspheres for protein adsorption, J. Chromatogr. B Anal. Technol. Biomed. Life Sci. 852 (2007) 62-68. doi:10.1016/j.jchromb.2006.12.044.

[27] J.B. Qu, G.S. Huan, Y.L. Chen, W.Q. Zhou, J.G. Liu, F. Huang, Coating gigaporous polystyrene microspheres with cross-linked poly(vinyl alcohol) hydrogel as a rapid protein chromatography matrix, ACS Appl. Mater. Interfaces. 6 (2014) 12752-12760. doi:10.1021/am5027067.

[28] A. Lamprou, Synthesis and application of novel polymeric materials: Short surfactants via ATRP and functional macroporous particles via Reactive Gelation under Shear, ETH Zurich, 2012.

[29] B. Brand, Macro-Porous chromatographic resins by controlled aggregation of colloid polymer particles, ETH Zurich, 2014.

[30] X.S. Chai, Q.X. Hou, F.J. Schork, Determination of residual monomer in polymer latex by full evaporation headspace gas chromatography, J. Chromatogr. A. 1040 (2004) 163-167. doi:10.1016/j.chroma.2004.04.024.

[31] C.M. Sorensen, Light Scattering by Fractal Aggregates: A Review, Aerosol Sci. Technol. Am. Assoc. Aerosol Res. 35 (2001) 648-687.

[32] A. Zaccone, D. Gentili, H. Wu, M. Morbidelli, Shear-induced reaction-limited aggregation kinetics of Brownian particles at arbitrary concentrations, J. Chem. Phys. J. Rheol. 132 (2010) 134903-673. doi:10.1122/1.551028. 


\section{4} 15 6

and Restructuring of Open and Dense Colloidal Aggregates, Langmuir. 27 (2011) 5739-5752. doi:10.1021/la1046589.

[34] S. Chow, Molecular Interpretation of the Glass Transition Temperature of PolymerDiluent Systems, Marcomolecules. 364 (1980) 362-364.

[35] P. Arosio, D. Xie, H. Wu, L. Braun, M. Morbidelli, Effect of Primary Particle Morphology on the Structure of Gels Formed in Intense Turbulent Shear, Langmuir. 26 (2010) 6643-6649. doi:10.1021/la9039754.

[36] J.K. Armstrong, R.B. Wenby, H.J. Meiselman, T.C. Fisher, The Hydrodynamic Radii of Macromolecules and Their Effect on Red Blood Cell Aggregation, Biophys. J. 87 (2004) 4259-4270. doi:10.1529/biophysj.104.047746.

[37] I. Nischang, Impact of biomolecule solute size on the transport and performance characteristics of analytical porous polymer monoliths, J. Chromatogr. A. 1354 (2014) 56-64. doi:10.1016/j.chroma.2014.05.053.

. 